\title{
La sobreeducación en España: estudio descriptivo y revisión crítica del concepto ${ }^{1}$
}

\section{Rafael Gobernado Arribas}

Universidad de Málaga. Departamento de Derecho del Estado y Sociología

Campus de El Ejido, s/n. 29071 Málaga

gobernado@uma.es

\section{Resumen}

Tradicionalmente, se ha observado la sobreeducación de diversas formas. Ninguna de ellas responde bien a la idea de sobreeducación. Para facilitar la tarea, hemos distinguido, primero, entre sobreeducación generalizada o consecuencia del cambio social hacia la modernidad, y sobreeducación relativa, que es la que nos interesa. Siempre hay sobreeducación relativa: afecta a una minoría dentro de cada categoría ocupacional. A su vez, hemos clasificado la relativa en dos: la relativa aparente y la relativa extrema o evidente. Solamente la sobreeducación relativa extrema (universitarios ocupados en las categorías laborales inferiores) es verdaderamente sobreeducación. Según los datos del año 2003, la población ocupada sobreeducada de acuerdo con este criterio apenas alcanzaba el 2,1 por ciento de la población laboral o el 3,2 de la población laboral joven.

Palabras clave: sobreeducación, mercado de trabajo, empleo, estatus social, ingresos.

\section{Abstract. Overeducation in Spain: description and critical review of the notion}

Overeducation has traditionally been studied in different ways, although none of them reflects the idea of overeducation correctly. In order to make our task easier, we have first distinguished between widespread overeducation, which is the consequence of social change towards modernity, and relative overeducation, which is the one we are interested in. Relative overeducation is always present and affects a minority of every occupational category (professional standing). In addition, we have also distinguished two kinds of relative overeduacation: debatable (unclear)and extreme or evident (obvious). Only the extreme kind (university graduates occupying a lower job) can strictly be called overeducation. According to this criterion, in 2003 the overeducated working population represented only a $2.1 \%$ of the general working population and a $3.2 \%$ of the young people who worked.

Key words: overeducation, labour market, employment, earnings, social status.

1. Este trabajo se ha realizado gracias a la colaboración del Centro de Estudios Andaluces: proyecto SOC-6, del año 2005. 


\section{Sumario}

1. Objetivos, antecedentes y tipología

2. Método, fuente y análisis de datos

3. La sobreeducación generalizada

4. La sobreeducación relativa
5. Sobreeducación subjetiva

6. Conclusiones

Bibliografía

\section{Objetivos, antecedentes y tipología}

Se entiende por sobreeducación el hecho de poseer un nivel educativo que excede las necesidades del puesto de trabajo. Sobreeducación es, así pues, sinónimo de infraocupación. Concretando algo más, se produce sobreeducación cuando el esfuerzo educativo no recibe suficientes compensaciones económicas ni sociales en el mercado laboral. Tal situación, en principio, es consecuencia del aumento del nivel educativo de la población demandante de empleo, mucho más acusado que el de las necesidades educativas o técnicas de la oferta de empleo. Aunque la idea general es fácil de definir, la operativización del concepto (su traducción a términos observables) no lo es tanto. Las fórmulas habituales de contabilizar la sobreeducación adolecen de falta de precisión y presentan resultados poco fiables. En el texto que sigue, se ofrece una descripción de la situación aludida en la población ocupada española, junto con una revisión crítica de la operativización del mismo. Es decir que, a la par que buscamos los datos en la población española ocupada, trataremos también de perfilar en lo posible los citados criterios de contabilidad. Para ello, se utilizan los datos de la Encuesta de calidad de vida en el trabajo, del Ministerio de Trabajo y Seguridad Social, tanto la del año 1999 como la del 2003.

El interés por la sobreeducación tiene ya su historia. En EE UU, los primeros estudios sobre el tema tuvieron lugar en los años sesenta. J. K. Folger y Ch. B. Nam (1964) tomaron nota de que la mayor parte del incremento de los requisitos educativos para ocupar empleos en las décadas de los cuarenta y los cincuenta en su país se debía al incremento del nivel educativo de la población empleada y no a las necesidades técnicas del puesto de trabajo. A una conclusión parecida llegó I. Berg (1970) con datos de los años cincuenta y sesenta. R. Rumberger (1981) hizo lo propio con datos posteriores, de 1960 a 1976. Desde entonces, se ha disparado la atención hacia la sobreeducación, y las publicaciones sobre este tema se han multiplicado, tanto en aquel país como en Europa. Un repaso detallado de los diferentes trabajos al respecto, tanto en Europa como en EE UU, se encuentra en P. J. Sloane (2003). Para un revisión de la sobreeducación en Europa, véase el resto de los trabajos incluidos en F. Büchel y otros (eds.) (2003).

También hay preocupación por el estudio de la sobreeducación en España. L. Garrido (1991) señaló, en su momento, que el sistema educativo español generaba más titulados superiores de los que necesita el sistema productivo. A. Alba-Ramírez (1993) fijaba, en 1985, una proporción de población ocu- 
pada sobreeducada del 17\%. C. García-Serrano y M. Malo-Ocaña (1996) entendían que tal población, en 1991, ascendía al 27\%. L. Fina Sanglas y otros (2000: 104) confirman la existencia de una «dualización de la estructura educativa de los españoles» en la década de los noventa: un exceso de titulaciones superiores y de inferiores, con un déficit de las intermedias, que son las que más necesita el sistema productivo.

El análisis de la sobreeducación parte de la premisa de que el mercado laboral y el sistema educativo están diseñados para ajustarse recíprocamente, por muy diferentes motivos según los modelos teóricos (véanse los siguientes resúmenes de tales modelos: M. J. García Espejo, 1998: 1-62; I. Brunet y A. Morell, 1998; R. Rubinson e I. Browne, 1994; S. M. Ruesga Benito y otros, 2000; R. Gobernado Arribas, 2003). Las razones del desajuste se encuentran, llegado el caso, en la propia interacción entre educación y empleo a través de la oferta y la demanda laboral. Así, el desajuste se debe fundamentalmente a que el nivel educativo se convierte en la principal señal para los empleadores a la hora de escoger candidatos para ocupar puestos laborales. Se produce, debido a ello, una espiral de crecimiento del nivel de estudios entre la población que busca empleo que no parece tener fin y que genera aún mayor desacoplamiento, ya que el incremento de las necesidades educativas de la oferta de empleo crecen mucho más despacio (R. Boudon, 1977; L. C. Thurow, 1977). Otras explicaciones de tipo menos general tratan también de justificar tamaño desajuste. Así, el paro empuja a aumentar el nivel educativo de los jóvenes, al generar el llamado "efecto guardería» (M. J. San Segundo Gómez y B. Petrongolo, 2000). Por su parte y también a título de ejemplo, desde el punto de vista de la teoría económica clásica, se ha pretendido explicar el desajuste por la falta de información del que se incorpora al mercado laboral (B. Jovanovic, 1979); desde el punto de vista de la teoría del capital humano, por la falta de la formación que se consigue en el propio puesto de trabajo (N. Sicherman y O. Galor, 1990). Incluso hay quien piensa que el propio desarrollo de la tecnología provoca la sobreeducación, ya que éste suele consistir en simplificar la mayor parte de las tareas laborales para la mayor parte de la población ocupada: cada vez hay que saber menos para hacer lo mismo (R. W. Rumberger, 1999).

De cualquier forma, estas explicaciones no son suficientes. La base del desajuste reside en que el sistema educativo es relativamente independiente del mercado laboral. Son muchos los estudios que han puesto de manifiesto otros intereses del sistema educativo que no son los laborales, igual como ocurre con los intereses políticos, religiosos, militares, etc. (véanse, por ejemplo, el clásico E. Durkheim, 1975; y los más recientes, J. Reis, 1993; D. C. North, 1982; R. Easterling, 1981) o propiamente institucionales (O. Ramírez y J. Boli, 1999; D. K. Muller, 1992; H. Steedman, 1992). La asignación al respectivo estrato social es también otra de las razones no económicas del sistema educativo: La segmentación educativa se guía no tanto por las necesidades laborales, como por mantener las diferencias de clase social (F. Ringer, 1992; R. Collins, 1989 o el clásico de Th. Veblen, 1974). Incluso es posible que la 
simple «búsqueda de la verdad» sea un motor importante de la educación (G. Snyders, 1978: 91 y s.). En último extremo, un repaso sobre los diversos orígenes de la escritura puede clarificar más ese entresijo de intereses (J. Goody, 1989). En resumen, el sistema educativo no tiene por qué coincidir con el mercado laboral ${ }^{2}$.

A los problemas teóricos anteriores relativos al ajuste entre educación y empleo, se deben añadir las dificultades técnicas de contabilidad de la población sobreeducada. Seguramente, estas dificultades tienen que ver con la deficiente teorización anterior, así como con la complejidad del tema. No obstante, los estudiosos utilizan diferentes modos para obtener información descriptiva, lo que origina, a su vez, diferentes resultados inevitablemente ${ }^{3}$. En general, hay tres maneras de acceder a la sobreeducación (P. J. Sloane, 2003: 15-20). Tales formas de contabilidad han sido desarrolladas y aplicadas sobre todo por economistas, lo que, sin lugar a dudas, sesga su operacionalización. Según la primera (medición objetiva), el investigador define la sobreeducación mediante escalas oficiales, previamente elaboradas por entidades públicas, que fijan el nivel educativo propio de cada categoría ocupacional. Se entiende que están sobreeducadas aquellas personas que superan tal nivel. Es el método más cómodo, pero en España no existe semejante catálogo oficial. Según la segunda (método empírico), el investigador saca por sí mismo el nivel medio educativo de cada categoría laboral y repite la tarea anterior: los que exceden de la media están sobreeducados. El problema, en este caso, es que el investigador suele trabajar, en general, con categorías agregadas, es decir, con categorías laborales muy amplias. A no ser que se utilicen las mismas categorías agregadas, los resultados serán diferentes. Otro inconveniente radica en determinar la cantidad de desviación sobre la media educativa para afirmar que existe sobreeducación. Se acepta que la distancia de una desviación estándar es suficiente.

La tercera manera (medición subjetiva) se basa en preguntar directamente al trabajador su opinión al respecto: «¿Diría Vd. que sus estudios son sufi-

2. En los análisis del mercado laboral, se suele establecer la relación entre educación y tal mercado mediante la argucia de añadir a la educación una categoría residual, el resto, lo no explicado. La adecuación se consigue dando por supuesto que existen otras variables que no se incorporan a la personalidad en el proceso de educación formal, sino fuera de él (habilidades comunicativas, manuales, inteligencia innata, redes sociales, formación en el empleo, etc.). C. Béduwé y J. Planas (2002: 50 y 51), en su estudio comparativo entre cinco países europeos sobre la relación entre la expansión educativa y el mercado de trabajo, utilizan la variable «años de experiencia» para ensamblar la oferta y la demanda de empleo, dando por sentado acertadamente que la mayoría de esas habilidades se ganan en el puesto de trabajo.

3. C. Béduwé y J. Planas (2002: 50 y 51), en vez de hablar de sobreeducación a secas, hablan de «frontera de competencia». Entienden por tal un límite formado por dos variables relacionadas: el nivel educativo y la experiencia profesional. Ambas se complementan, cuanta más experiencia, menos titulación académica se necesita para alcanzar la misma frontera de competencia. Las personas que se encuentren más allá de esa frontera, forman la población «sobreeducada». 
cientes para realizar su trabajo actual?». Este método subjetivo se completa con información, también subjetiva, sobre la suficiencia de formación (conocimientos adquiridos en el puesto de trabajo) y sobre la adecuación de los conocimientos a las necesidades del puesto de trabajo (si, por ejemplo, siendo licenciado en Ciencias Químicas se dan clases de Física). El método subjetivo es el seguido por A. Alba-Ramírez y M. Benítez (2003) al estudiar la sobreeducación en España recientemente.

Como ya se anunció arriba, no parece existir coincidencia entre los tres diferentes métodos (el objetivo, el subjetivo y el empírico). Cada uno da unos resultados distintos. La correlación entre las diferentes medidas es escasa (H. Battu y otros, 2000).

Nuestro punto de partida consiste en reconocer la existencia de dos formas de entender la sobreeducación que exigen un tratamiento muy diferente: la sobreeducación generalizada y la sobreeducación relativa. Se entiende por la generalizada aquélla que alcanza a la totalidad (o a la gran mayoría) de la población. En la medida que la expansión educativa alcanza a más población (expansión horizontal) y que cada vez se estudia durante más años (expansión vertical), la sobreeducación termina por afectar a todos. No es problemática, ya que se termina por ver como "normal». Su análisis, en último extremo, se enmarca en el ámbito del cambio social, la modernización y el desarrollo económico. Por su parte, la sobreeducación relativa es aquélla que afecta solamente a una minoría de cada categoría ocupacional: unos pocos tienen un nivel educativo superior al resto de la categoría en cuestión. Este tipo es el que ha interesado sobre todo a sociólogos y economistas.

Por supuesto, ambos tipos están totalmente relacionados: los procesos de expansión (en este caso, de expansión educativa) siguen una pauta en forma de «S» que consiste en afectar, primero, a una minoría para, después, crecer hasta que alcanza al resto de la población (en este caso, al resto de la población de la categoría ocupacional). De cualquier forma, ambos tipos, la generalizada y la relativa, deben ser considerados más un proceso que una situación estable. En ambos casos, también, se trata siempre de conceptos relativos: la sobreeducación en un momento y lugar sólo puede entenderse en comparación con otra situación (diacrónica o sincrónica) del mercado de trabajo. No existe una relación natural ni objetiva entre el nivel educativo y la categoría ocupacional.

La sobreeducación relativa se diferencia, no obstante, de la generalizada en que puede ser más problemática que ésta, ya que la comparación entre la minoría y el resto de la categoría ocupacional puede dar lugar a frustaciones de diverso tipo.

Para facilitar la operacionalización de la sobreeducación relativa, hemos diferenciado también dos tipos de ésta: la sobreeducación relativa evidente o extrema y la sobreeducación relativa aparente, discutible. La evidente es aquélla que se produce entre los niveles extremos de educación (universitarios) y de prestigio ocupacional (estrato inferior). Es decir, aquélla en la que no hay duda de que es real. Por el contrario, la sobreeducación aparente siempre alber- 
gará la duda de su propia existencia. Las tres fórmulas propuestas arriba (medición objetiva, empírica y subjetiva) encajan en nuestra categoría de aparentes. La prueba básica para detectar la sobreeducación relativa, del tipo que sea, es la igualdad de ingresos entre los sobreeducados y el resto de población de la categoría ocupacional pertinente. No hay sobreeducación si ésta sirve para obtener más ingresos que los demás en la misma categoría de puesto de trabajo.

\section{Método, fuente y análisis de datos}

La base de datos de este trabajo lo constituye la Encuesta de calidad de vida en el trabajo del año 1999 y la del 2003, realizadas ambas por el Ministerio de Trabajo y Seguridad Social durante los meses de junio. Cada encuesta se realiza sobre una muestra de seis mil personas ocupadas. La muestra ha sido ponderada por los propios autores según sexo, edad, comunidad autónoma y tamaño del municipio. Ha de entenderse como una muestra altamente representativa de la población ocupada española de esos años.

La variable de más interés en nuestro análisis es el «nivel educativo del entrevistado». A partir de la información del cuestionario, se ha reelaborado esta variable en otras dos: «años de escolaridad» y «nivel educativo». Los «años de escolaridad» se han calculado según los años oficiales en que se tarda en obtener el título educativo. Se ha aplicado un criterio que ya es habitual (por ejemplo J. Carabaña, 1999: 282-283). Así, los resultados varían desde 0, los analfabetos, hasta 20 años los que son doctores o tienen estudios de postgrado. Por su parte, la variable "nivel educativo» está formada por cuatro categorías (o valores de la variable): analfabetos y sin estudios, estudios primarios, estudios secundarios y estudios universitarios. Se prescinde de la categoría de analfabetos y sin estudios en una oportunidad del análisis que sigue: cuando se analiza el peso de la educación en la organización, entonces no tiene sentido incluir el valor nulo de esa categoría.

A partir de la información sobre la "ocupación del entrevistado», hemos formado dos variables nuevas: la «escala de prestigio ocupacional» (continua o cuantitativa) y los "estratos ocupacionales» (discontinua o cualitativa). Para crear la escala indicada, se ha aplicado la Escala de prestigio ocupacional elaborada por J. Carabaña y C. Bueno (1996) a los datos sobre ocupación de las encuestas. Es decir, se han ordenado las ocupaciones declaradas por los entrevistados según tal escala. Una vez conseguida la primera variable, se pasó a la segunda: «los estratos ocupacionales». El continuo de prestigio formado con la escala se dividió arbitrariamente en cinco categorías o estratos atendiendo al criterio poblacional: cada estrato debía tener aproximadamente un quinto de la población muestral. Las etiquetas de los estratos son de tipo relacional: alto, medio-alto, medio, medio-bajo y bajo.

A su vez, la variable continua «edad» se ha recodificado en otra discreta o cualitativa: "grupos de edad». Ésta tiene tres categorías (o valores): jóvenes (menores de 30 años), adultos (de 30 a 50 años) y mayores (más de 50 años). 
Por su parte, se ha transformado los «ingresos» en una variable continua, asignando a cada categoría (intervalo) el valor de la marca de clase correspondiente. Los datos del cuestionario de 1999 se han traducido a euros, siguiendo el criterio explícito en el cuestionario de 2003.

Para precisar la relación entre variables, se han utilizado dos tipos de coeficiente: el coeficiente de contingencia y la prueba de la F. El primero se utiliza para establecer grados de relación entre variables cualitativas. Se completa con el coeficiente V de Cramer para facilitar la lectura del coeficiente de contingencia. La prueba de la $\mathrm{F}$ es un criterio de significación de relación entre variables cuando la variable independiente es cualitativa y la dependiente es cuantitativa. Consiste, en resumen, en comparar la varianza entre grupos y la varianza intragrupos (véase J. J. Sánchez Carrión, 1995: 339-341). Como medida de dispersión, se ha utilizado el coeficiente de variación (c. v.) o porcentaje de la desviación estándar sobre la media.

A lo largo del análisis, se ha utilizado la variable continua «ingresos» como elemento de prueba de la bondad de la definición de "población sobreeducada». Se da por supuesto que la población sobreeducada real no puede tener ingresos superiores al resto de la población ocupada. En caso contrario, los sobreeducados no serían tales, ya que estarían rentabilizando sus estudios.

\section{La sobreeducación generalizada}

Hay dos formas de acceder a la sobreeducación generalizada: en un sentido amplio, enfocando el tema desde la teoría del cambio social, y, en su sentido estricto, enfocándolo desde el punto de vista de la relación entre educación y ocupación.

En su sentido estricto, es muy difícil medir la sobreeducación generalizada. El incremento de la escolarización de la población ocupada es obvio. Lo que es más complicado es detectar el nivel educativo óptimo para cada ocupación en un momento determinado. Se esgrimen dos razones por las que pueden aumentar las exigencias educativas de la estructura laboral: en primer lugar, porque aumentan las posiciones de orden técnico o de nivel superior que se da por supuesto que necesitan un nivel educativo alto; en segundo lugar, porque la mayoría de las posiciones sociales, tanto las altas como las bajas, necesita más conocimientos para su desempeño a medida que se desarrolla la tecnología. Mientras que es fácil controlar la primera causa de variación, en nuestro caso mediante la escala de prestigio ocupacional, no lo es tanto la segunda. La escala de prestigio ocupacional puede servir, sin embargo, de orientación de las necesidades técnicas y educativas de cada categoría laboral. Así pues, el exceso de crecimiento de la media de años de escolaridad sobre la media de prestigio ocupacional es un indicador aproximado de sobreeducación generalizada.

De los datos de la tabla 1 se desprende que el incremento educativo de la población ocupada española ha sido muy superior al de la creación de puestos altos del sistema laboral. Se aprecia, incluso, que la estructura laboral ha evolucionado en esos cuatro años hacia posiciones más bajas y presumible- 
Tabla 1. Medias y coeficientes de variación de prestigio ocupacional y de años de escolaridad, años 1999 y 2003, en la población española ocupada.

\begin{tabular}{lcclllc}
\hline & \multicolumn{2}{c}{1999} & & \multicolumn{2}{c}{2003} & \\
\cline { 2 - 3 } & Media & c. v. & & media & c. v. & Variación: $\mathbf{1 9 9 9 = 1 0 0}$ \\
\hline Prestigio & 106,1 & $34,5 \%$ & & 104,6 & $35,1 \%$ & 98,6 \\
\hline Escolaridad & 9,67 & $46,5 \%$ & & 10 & $41 \%$ & 103,4 \\
\hline
\end{tabular}

Fuente: Encuesta de calidad de vida en el trabajo, 1999 y 2003. Elaboración propia.

mente con menores exigencias educativas. Mientras que la media de años de escolaridad sube de 9,67 años a 10, la media de prestigio ocupacional desciende desde 106,1 a 104,6. En resumen, en el 2003 había que estudiar más para obtener una ocupación peor considerada que en el 1999.

Para facilitar la comparación, hemos sintetizado la información anterior en un indice de sobreeducación generalizada. Tal índice se define como la diferencia (distancia) entre la variación porcentual de la media de años de escolaridad (103,4 de la tabla 1$)$ y la de prestigio ocupacional $(98,6$ de la misma tabla 1). (El valor del índice en el ejemplo es 4,8.) Los datos de este índice se recogen en la tabla 2 . De esta tabla, se desprende que el mayor incremento educativo lo sufrieron los padres de los entrevistados del 2003 (los resultados son muy similares a los datos del 1999). El índice correspondiente de sobreeducación generalizada asciende a 75,2. La expansión educativa tuvo lugar en España fundamentalmente a partir de la aplicación de la Ley de Educación de Villar Palasí de 1970 (R. Gobernado, 1996). Es entonces cuando se deja de considerar la educación como consumo y se pasa a considerarla como inversión (INE, 1994: 153). El cambio fue, obviamente, más educativo en la población ocupada que técnico en la estructura ocupacional. No obstante, se ha de tener en cuenta también el cambio tecnológico reciente que ha tenido lugar a partir de 1980 (M. Castells, 2000). De cualquier forma, se aprecia el gran desajuste entre la estructura ocupacional y la escolaridad de la población en el cambio social ocurrido entre las dos generaciones indicadas.

Observando los cambios habidos en los cuatro años que median entre ambas encuestas (1999-2003), se aprecia que sigue aumentando la escolarización por encima de la media de prestigio ocupacional (índice de sobreeducación generalizada: 4,8), si bien lo hace a un paso más lento que en la comparación intergeneracional. Por su parte, en estos cuatro años, las mujeres han sentido más abundantemente la sobreeducación que los varones, casi el doble. Y entre éstas, han sido las de mayor edad las que han sufrido una mayor sobreeducación $(14,2)$. Por el contrario, las jóvenes son las que la experimentan en menor medida, menos incluso que cualquier categoría de varones.

Sin embargo, por muy claros que sean los datos anteriores, queda la duda sobre si la estructura ocupacional en general necesitaba población con más años de escolaridad o no la necesitaba. De ahí el interés del punto de vista del cambio social, que no entra en esos temas tan concretos. Desde este punto de 
Tabla 2. Índice de sobreeducación generalizada (distancia entre la variación de la media del prestigio ocupacional y la de los años de escolaridad) según sexo y edad.

\begin{tabular}{|c|c|c|}
\hline Padres / entrevist. 2003 & Total & $\begin{array}{c}\text { Distancia } \\
75,2\end{array}$ \\
\hline \multirow[t]{3}{*}{1999 / 2003} & Total & 4,8 \\
\hline & Varones & 3,6 \\
\hline & Mujeres & 6,2 \\
\hline \multirow[t]{3}{*}{ Varones } & Jóvenes & 5,0 \\
\hline & Adultos & 3,5 \\
\hline & Mayores & 5,2 \\
\hline \multirow[t]{3}{*}{ Mujeres } & Jóvenes & 2,9 \\
\hline & Adultas & 7,1 \\
\hline & Mayores & 14,2 \\
\hline
\end{tabular}

Fuente: Encuesta de calidad de vida en el trabajo, 1999 y 2003. Elaboración propia.

vista, más general, la sobreeducación es una consecuencia de la expansión educativa, la cual, a su vez, forma parte del proceso más general de modernización y desarrollo económico. En este proceso, hay un rasgo que nos interesa: las categorías sociales cada vez están menos relacionadas entre sí. Así, por ejemplo, la categoría ocupacional del padre cada vez tiene que ver menos con la del hijo; la clase social cada vez explica menos la dirección de voto o la práctica religiosa, etc. La movilidad social, la movilidad espacial, los medios de comunicación de masas, la educación universal, entre otros elementos, ayudan a explicar este cambio. El proceso recibe el nombre de individuación, ya que, al final, el individuo toma decisiones con independencia de sus orígenes y de sus logros.

En el caso que nos ocupa, hemos considerado tres variables: los «años de escolaridad», el "prestigio ocupacional» y los «ingresos». De acuerdo con la idea anterior, estas tres variables estarían muy relacionadas en una sociedad tradicional y escasamente relacionadas en una sociedad moderna. Definimos la sobreeducación generalizada, por lo tanto, como el resultado de un doble proceso: el de expansión educativa y el de autonomía de la educación respecto a las otras dos variables (prestigio ocupacional e ingresos). Ambas evoluciones van relacionadas.

En España, ha aumentado el nivel de ingresos de la población. Los datos que barajamos — son datos de la propia encuesta - informan que se ha pasado de 933 euros de media por mes y entrevistado en el año 1999 a 1.057 en el 2003. Esto supone que los ingresos medios en el 2003 son el 113,3 por ciento de los del 1999. Este cálculo es muy burdo, ya que no tiene en cuenta la inflación real, muy alta con motivo del cambio de pesetas a euros. No obstante, el dato es un indicativo de la mejora de renta. Hay, sin embargo, otra variación que tiene ahora más interés: en esos cuatro años ha disminuido la diferencia de 
ingreso entre los distintos niveles educativos. O lo que es igual, la educación cada vez explica menos las diferencias de ingresos. Mientras que, en 1999, el nivel educativo («sin estudios», "primarios», «secundarios» y "superiores») tenía mucho que ver con las medias de ingresos $(\mathrm{F}=201166,4$; sign. $=0,000)$, cuatro años más tarde, esa relación decae profundamente $(\mathrm{F}=159,3$; sig. $=0,000)$. No se trata de ninguna sorpresa, semejante proceso se había observado ya tiempo atrás (L. C. Thurow, 1977; R. Gobernado, 1996). Parece lógico que, si aumenta más deprisa el nivel educativo que los ingresos, éstos se independicen cada vez más de aquél. Pero, volviendo a nuestro tema: la relativa independencia de los ingresos respecto al nivel educativo se constituye, asi pues, en otro indicador de sobreeducación general.

De acuerdo con el punto de vista del cambio social, la sobreeducación se diluye y se compensa con los otros procesos sociales, en nuestro caso: la expansión educativa, el cambio de la estructura ocupacional y la evolución de los ingresos. De esta forma, la sobreeducación se integra en el paisaje social y cultural como una consecuencia esperada y espontánea del proceso de modernización. Ésta es otra de las razones de la dificultad de captar la sobreeducación en la realidad (tanto la generalizada como la relativa), en otras palabras, de la dificultad de operativizar el concepto.

\section{La sobreeducación relativa}

\section{A) Sobreeducación relativa aparente}

Un primer intento, fallido, de contabilizar este tipo de sobreeducación en el presente trabajo se ha efectuado siguiendo el llamado arriba "criterio empírico». Para ello, se ha clasificado la población ocupada en cinco estratos según la escala de prestigio ocupacional. La población de cada estrato supone aproximadamente el 20 por ciento de la población encuestada. A su vez, la población de cada estrato ha sido distribuida en tres categorías según el número de años de escolaridad: población sobreeducada (una desviación estándar por encima de la media de años de escolaridad); población infraeducada (una desviación estándar por debajo de la media), y población ajustada (el resto).

Según los datos de la tabla 3, la población sobreeducada calculada a partir de los estratos de prestigio ocupacional ${ }^{4}$ alcanza al 16,4 por ciento de la pobla-

4. Cualquier clasificación de las ocupaciones puede servir de criterio para marcar la sobreeducación. Así, por ejemplo, la clasificación en las cinco clases ocupacionales (directivos, técnicos, empleados, manuales calificados y manuales sin calificar). Los resultados ahora discrepan algo de los anteriores. La población sobreeducada en este caso alcanza al 15,1 por ciento (con el criterio anterior era el 16,4), la población ajustada es el 74,5 (con el criterio anterior, el 66,7) y la población infraeducada es el 10,4 (con el criterio anterior, el 16,8). La clase con mayor proporción de población sobreeducada es la de "manuales calificados» $(19,6)$. La que menos proporción tiene es la de «técnicos» $(5,3)$. Según este criterio, la población sobreeducada femenina (14 por ciento) es algo menor que la masculina $(15,9)$. La edad presenta la misma tendencia que antes: la población está más sobreeducada cuanto más joven es. 
Tabla 3. Población ocupada española sobreeducada, infraeducada y ajustada, según estratos de prestigio ocupacional

\begin{tabular}{lcccll}
\hline Estrato & Infraeducados & Ajustados & Sobreeducados & Total & $N$ \\
\hline Alto & 16,8 & 75,9 & 7,4 & 100 & 1.172 \\
Medio alto & 16 & 60,4 & 23,7 & 100 & 1.144 \\
Medio & 13,5 & 75,7 & 10,7 & 100 & 1.186 \\
Medio bajo & 29,7 & 45,9 & 24,4 & 100 & 1.025 \\
Bajo & 10,8 & 72 & 17,2 & 100 & 1.352 \\
\hline Total & 16,8 & 66,7 & 16,4 & 100 & 4.527 \\
\hline
\end{tabular}

Fuente: Encuesta de calidad de vida en el trabajo, 2003. Elaboración propia.

ción ocupada en España. Se encuentra infraeducado el 16,8 por ciento. Los datos de cada uno de los estratos varían mucho: desde el 7,4 por ciento del estrato superior hasta el 24,4 del estrato medio bajo. Estos datos pertenecen a la encuesta del año 2003, pero apenas difieren de los resultados de la encuesta del 1999. Incluso los sobreeducados, en general, son algo más en este año (16,6 por ciento) que en el $2003(16,4)$. La variación se aprecia algo más en la proporción de infraeducados: el 18,9 por ciento en el 1999 y el 16,8 cuatro años después. La adaptación se está consiguiendo reduciendo el número de infraeducados, resto de una economía tradicional.

De los datos de la tabla 4 se desprende que la población sobreeducada femenina $(21,3$ por ciento) es superior a la masculina $(13,4)$. También se aprecia, en la misma tabla, que, cuanto más aumenta la edad, más disminuye la proporción de población sobreeducada: los jóvenes presentan porcentajes de sobreeducación más altos que los adultos y éstos, a su vez, más altos

Ajuste educativo de los estratos de prestigio ocupacional según el número de clases ocupacionales. Porcentajes sobre el total

\begin{tabular}{lcccc}
\hline Ajuste educativo & \multicolumn{4}{c}{ Ajuste educativo según clase ocupacional } \\
según estrato & Infraeducados & Ajustados & Sobreeducados & Total \\
\hline Infraeducados & 9 & 7,9 & 0 & 16,9 \\
Ajustados & 1,4 & 61,6 & 4 & 66,9 \\
Sobreeducados & 0 & 5 & 11,1 & 16,2 \\
\hline Total & 74,5 & 10,4 & 15,2 & 100 \\
\hline
\end{tabular}

Coeficiente de contingencia: 0,671 (sig. =0,000). V de Cramer: 0,639.

Fuente: Encuesta de calidad de vida en el trabajo, 2003. Elaboración propia.

De cualquier forma, se aprecia una aceptable correspondencia entre la población de ambos criterios, tal y como se aprecia en la tabla de esta nota. El 81,7 por ciento de la población observada coincide en ambas clasificaciones. En otras palabras, el coeficiente V de Cramer (que oscila entre 0 y 1 ) es relativamente alto. Y esto no se debe sólo a que exista cierto solapamiento entre las dos clasificaciones ocupacionales, la de estratos y la de clases ocupacionales, ya que el mismo coeficiente en este caso es ligeramente menor (V de Cramer: $0,574)$. En resumen, en principio cualquiera de las dos clasificaciones puede servir. 
Tabla 4. Población ocupada española sobreeducada, infraeducada y ajustada, según sexo y edad, año 2003.

\begin{tabular}{llcccc}
\hline & & Infraeducados & Ajustados & Sobreeducados & Total \\
\hline Varones & Total & 20,4 & 66,1 & 13,4 & 100 \\
& Jóvenes & 8,7 & 74,7 & 16,6 & 100 \\
& Adultos & 18,1 & 67,5 & 14,5 & 100 \\
& Mayores & 40,9 & 52,3 & 6,8 & 100 \\
\hline Mujeres & Total & 11 & 67,7 & 21,3 & 100 \\
& Jóvenes & 4,7 & 65,8 & 29,4 & 100 \\
& Adultas & 9,4 & 70,2 & 20,5 & 100 \\
& Mayores & 30 & 61,8 & 8,3 & 100 \\
\hline
\end{tabular}

Fuente: Encuesta de calidad de vida en el trabajo, 2003. Elaboración propia.

también que los mayores. No se puede inferir de estos datos, no obstante, si se trata de una situación generacional (propia de las cohortes) o propia del ciclo profesional.

Esta fórmula es la más usual para calcular la sobreeducación: el criterio empírico, tal y como se dijo arriba. Sin embargo, no es un criterio convincente de selección de la población sobreeducada. Los ingresos declarados por los entrevistados varían siempre a favor de la población sobreeducada. En otras palabras, no hay tal sobreeducación si el nivel educativo alto sirve para ganar más dinero, aún perteneciendo al mismo estrato ocupacional. Los datos de la tabla 5 son claros: es rentable tener un nivel educativo superior a una desviación estándar sobre la media de cada categoría ocupacional. Las diferencias entre las medias de ingreso a favor de los sobreeducados se repiten en cada uno de los cinco estratos ocupacionales considerados. Y así ocurre en la mayor parte de los estudios de sobreeducación (S. Rubb, 2003; C. Béduwé y J. Planas, 2002: 54): es rentable tener un nivel educativo superior al resto.

Tabla 5. Media de ingresos (en euros) mensuales y coeficiente de variación según ajuste educativo por estratos. Población ocupada española, año 2003.

\begin{tabular}{lllr}
\hline Adecuación & Media & c. v. & \multicolumn{1}{c}{} \\
\hline Infraeducados & 1.008 & $49,2 \%$ & 744 \\
Ajustados & 1.045 & $47,8 \%$ & 3.217 \\
Sobreeducados & 1.106 & $60,4 \%$ & 813 \\
Total & 1.050 & $50,8 \%$ & 4.774 \\
\hline
\end{tabular}

Coeficientes: $\mathrm{F}=7015,6$ (sig. $=0,000$ ).

Fuente: Encuesta de calidad de vida en el trabajo, 2003. Elaboración propia. 
Algo parecido ocurre con los datos de 1999: la población sobreeducada ingresa muchos más euros que la población ajustada, la cual, a su vez, tiene unos ingresos superiores a la población infraeducada. La particularidad reside en que la relación entre el ajuste educativo y los ingresos es mayor en los datos del año $1999(\mathrm{~F}=20.201,5$; sig. = 0,000) que en los datos del 2003 $(\mathrm{F}=7.015,6$; sig. $=0,000)$. O lo que es igual, el nivel educativo es más rentable en 1999 que después.

Volvemos a nuestro objetivo. Para tratar de solventar ese problema en la medición de la sobreeducación, se utiliza ahora una tercera variable que sirve de elemento corrector: la edad del entrevistado. Entre los «jóvenes», la media de ingresos de los que desempeñan una ocupación ajustada a su nivel educativo es acusadamente mayor que la de los sobreeducados. Esto no ocurre con los «adultos» ni con los «mayores», entre los que un mayor nivel educativo es motivo de mayores ingresos, aun dentro de la misma categoría ocupacional. De estos datos parece inferirse que la contabilidad de la población sobreeducada debe completarse con la edad del entrevistado. Más concretamente, entre la población joven el criterio empírico de detección de sobreeducación aparenta relativa validez. No lo es entre la población ocupada adulta ni entre la mayor. El interés puede ser sólo aparente, ya que la experiencia (tiempo trabajando) es un factor muy importante de rentabilizar el título educativo, como ya se sabe (R. Gobernado, 2003: 24). Según esto, la sobreeducación se debe apreciar en la carrera profesional global, no en los inicios de la misma, tal y como sugiere la noción de "competencia» de C. Béduwé y J. Planas (2002: 50 y 51). Ahora bien, sólo manejamos datos sincrónicos y de ellos no se puede desprender ningún análisis diacrónico serio. No obstante, a juzgar por la abundante expansión educativa reciente en España, hay muchas posibilidades de que la situación de la cohorte joven se prolongue más allá de la juventud, lo que da lugar a una nueva forma de organización del mercado laboral basada precisamente en la sobreeducación.

Un segundo intento, también fallido, de contar la población sobreeducada se ha seguido clasificando la población según las dos variables siguientes: primero, el nivel educativo (tres niveles: estudios primarios, secundarios y superiores) y, segundo, el lugar que se ocupa en la organización (directivo, supervisor y empleado). Se supone que cuanto más alto sea el lugar que se ocupa en el organigrama, mayor será también el nivel educativo. En la tabla 6 se cruzan ambas variables. La relación entre las dos variables es alta, a juzgar por el coeficiente de contingencia.

Los valores de la diagonal corresponden teóricamente a la población cuyos niveles educativos concuerdan con la tarea en la organización. Los valores que están por encima de la diagonal son los de la población infraeducada. Los de debajo de la diagonal son los sobreeducados. Según esto, está sobreeducado el 66,2 por ciento de la población entrevistada. Con niveles educativos ajustados, el 25,5 por ciento. La población infraeducada se queda en el 8,3 por ciento. Las variaciones en la estructura educativa y en la organizativa, que se escapan de este trabajo, hacen que las diferencias de esos datos con los de 1999 
Tabla 6. Nivel de estudios según posición en la organización de la población ocupada española del 2003. Porcentajes sobre el total.

\begin{tabular}{lcccr}
\hline & \multicolumn{4}{l}{ Nivel de estudios } \\
\cline { 2 - 5 } Posición en la organización & Superiores & Secundarios & Primarios & Total \\
\hline Dirección & 2,6 & 4,4 & 2,2 & 9,2 \\
\hline Supervisión & 4,1 & 6,9 & 1,7 & 12,7 \\
\hline Empleado & 13,9 & 48,2 & 16 & 78,1 \\
\hline Total & 20,7 & 59,5 & 19,9 & 100 \\
\hline
\end{tabular}

Coeficiente de contingencia $=0,671$ (sig. $=0,000)$; $\mathrm{V}$ de Cramer $=0,639 . \quad$ N: 5.436 .

Fuente: Encuesta de calidad de vida en el trabajo, 2003. Elaboración propia.

sean muy acusadas: la población sobreeducada en 1999 alcanzaba el 35,4 por ciento (menos que cuatro años después); la población ajustada llegaba al 49,4 (casi el doble que más tarde) y la infraeducada era el 15,2 (también casi el doble que en el año 2003).

El cálculo anterior tiene la ventaja de su facilidad. Sin embargo, este criterio no parece más útil que el anterior. En principio, se aprecia que el grado de ajuste de la educación apenas tiene que ver con la media de ingresos, incluso opera al revés que antes. Así, la media de ingresos de los infraeducados es superior a la de los demás ocupados. Sin embargo, esto no es ahora indicativo de bondad del criterio, sino que se debe a que los infraeducados ocupan las dos posiciones más altas en la organización (dirección y supervisión); mientras que los sobreeducados ocupan las dos posiciones inferiores (supervisión y empleado): Véase la tabla 6. Hay que tener en cuenta que la posición en la organización es la que determina en mayor proporción los ingresos, mucho más que el nivel educativo (R. Gobernado, 2003: 16 y 17).

La edad opera ahora de forma inversa que antes. Recordemos que el lugar en la organización explica más el monto de ingresos, que el nivel de estudios. En la población joven apenas hay diferencia entre la media de ingresos de los infraeducados y la de los sobreeducados: por supuesto que los ingresos de los infraeducados son superiores, pero no mucho $(F=4.932,3$; sig. $=0,000)$. En la población adulta, esa distancia es bastante más acusada: lo que ganan los infraeducados es mucho más que lo que ganan los sobreeducados $(\mathrm{F}=21.000,9$; sig. $=0,000)$. Esta diferencia se mantiene en la población mayor. Todo esto se explica más por el lugar que se ocupa en la organización (los jóvenes ocupan posiciones inferiores y los adultos, posiciones superiores) que por el nivel educativo (los jóvenes tienen un nivel educativo alto y los adultos, bajo).

La relación entre ambas formas de contabilizar la población sobreeducada es reducida. Sólo coincide el 31 por ciento de los encuestados. La población sobreeducada, de acuerdo con ambos criterios, es nada más un 15 por ciento. 
Tabla 7. Media de ingresos (en euros) mensuales y coeficiente de variación según ajuste educativo en la organización. Población ocupada española, año 2003.

\begin{tabular}{lrlr}
\hline Adecuación: & Media & c. v. & $N$ \\
\hline Infraeducados & 1.313 & $48 \%$ & 319 \\
Ajustados & 1.083 & $57,5 \%$ & 1.127 \\
Sobreeducados & 999 & $45,4 \%$ & 3.066 \\
Total & 1.042 & $50,1 \%$ & 4.512 \\
\hline
\end{tabular}

Coeficientes: $\mathrm{F}=58378,4$ (sig. $=0,000$ ).

Fuente: Encuesta de calidad de vida en el trabajo, 2003. Elaboración propia.

Tabla 8. Ajustes educativos según el criterio de posición en la organización y según la posición en la organización. Porcentajes sobre el total.

\begin{tabular}{|c|c|c|c|c|}
\hline Ajuste educativo & Ajuste educativ & según estra & ocupacional & \\
\hline según organización & Infraeducados & Ajustados & Sobreeducados & Total \\
\hline Infraeducados & 4,1 & 4 & 0,2 & 8,3 \\
\hline Ajustados & 10,7 & 13 & 1,7 & 25,5 \\
\hline Sobreeducados & 0,9 & 50,5 & 14,9 & 66,2 \\
\hline Total & 15,7 & 67,4 & 16,9 & 100 \\
\hline
\end{tabular}

Coeficiente de contingencia $=0,493$ (sig. $=0,000$ ). $\mathrm{V}$ de Cramer $=0,401 . N=5.371$.

Fuente: Encuesta de calidad de vida en el trabajo, 2003. Elaboración propia.

\section{B) Sobreeducación relativa evidente}

Según un primer criterio, se consideran sobreeducados de forma evidente aquellos entrevistados de los estratos bajos (medio-bajo y bajo) que tienen estudios superiores (diplomaturas o licenciaturas). Este criterio parece ser válido. En total, hay 122 entrevistados en esas condiciones, lo que supone el 2,1 por ciento de toda la muestra y el 5,1 de la población de los estratos bajos. Así mismo, supone el 3,2 por ciento de la población joven, el 2 por ciento de la población adulta y el 1 de la población mayor. Verdaderamente, la sobreeducación evidente es muy reducida. Esto parece ocurrir en los cinco países estudiados por C. Béduwé y J. Planas (2002: 52), donde apenas se puede hablar de sobreeducación acusada.

Como era de suponer, esta clasificación cumple el requisito de los ingresos: apenas hay diferencia entre los ingresos de los universitarios de los estratos bajos y de los no universitarios de los mismos estratos $(\mathrm{F}=0,38$; sign. $=0,535)$. La población joven universitaria tiene, incluso, unos ingresos menores que la no universitaria, aunque dentro de unas diferencias míninas $(\mathrm{F}=3,644$; sig. $=0,057)$. En la población adulta, los universitarios tienen unos ingresos 
algo superiores, pero sin excesiva diferencia $(F=4,706$; sign. $=0,030)$. En la población mayor, no hay tanta diferencia de ingresos como en la de adultos ${ }^{5}$.

Según un segundo criterio, es sobreeducada la población ocupada en el nivel de «empleados» y que tienen título educativo universitario (diplomatura o licenciatura). Según esto, es sobreeducado el 13,7 por ciento de la población entrevistada. Sin embargo, el cálculo de la sobreeducación evidente con tal definición no pasa la prueba de los ingresos. Sin embargo, los empleados universitarios tienen unos ingresos muy superiores a los que no son universitarios $(\mathrm{F}=228,3$; sign. $=0,000)$.

Es obvio que el indicador más preciso de sobreeducación relativa es el de sobreeducación evidente elaborado a partir de los estratos sociales bajos y el nivel educativo universitario. Falta por explicar, sin embargo, por qué los resultados son tan exiguos. Parece difícil asumir que solamente haya un 2,1 por ciento de población sobreeducada. La respuesta se encuentra al comparar los datos del año 1999 con los del 2003. En primer lugar, este tipo de sobreeducación entonces era todavía menor (1,5 por ciento). Sin embargo, esta minoría universitaria que ocupaba los estratos inferiores de la estructura de prestigio ocupacional tenía unos ingresos muy superiores a los demás miembros de esos estratos inferiores $(F=6.057,8$; sig. $=0,000)$. Es decir, todavía en 1999 era rentable invertir en educación, independientemente de la ocupación. Cuatro años después, ya ha desaparecido esa rentabilidad, al menos en esos estratos ocupacionales. Esto significa que la sobreeducación es absorbida fácilmente por la estructura ocupacional, incluso aunque el nivel medio de prestigio descienda algo. Un mecanismo del que se sirve el sistema socioeconómico para compensar el exceso educativo es el de reducir el interés económico del esfuerzo educativo: las desigualdades educativas explican menos las diferencias de ingresos en el 2003 que en el 1999. Las variaciones de la estructura ocupacional, del nivel de ingresos y del nivel educativo se compensan y se reequilibran en un proceso de continua búsqueda de equilibrio del sistema.

\section{Sobreeducación subjetiva}

En el cuestionario de la Encuesta de calidad de vida en el trabajo de 1999, se hace la siguiente pregunta ( $\left.\mathrm{n}^{\circ} 106\right)$ : «¿Diría Vd. que sus estudios son suficientes para realizar su trabajo actual?». Se dan tres opciones de respuesta: más que suficientes, suficientes e insuficientes. La respuesta a esta pregunta es subjetiva, depende de la opinión del entrevistado. Es otro indicador de sobree-

5. Los resultados son muy parecidos si se utiliza el criterio de clase manual ocupacional (cualificada y sin cualificar). El total de sobreeducados suma 104 entrevistados, es decir, el 1,7 por ciento de la muestra y el 3,6 de la población de la clase ocupacional manual. La media de ingresos entre ambas categorías (los universitarios y los no universitarios) coincide también $(\mathrm{F}=0,175$; sign. $=0,676)$. Coinciden plenamente ambos criterios, el que se basa en las clases ocuapcionales y el de los estratos: los 104 sobreeducados de la clase manual también lo son según el criterio del estrato social bajo. 
ducación. Esta pregunta no se ha repetido en el cuestionario del 2003, por lo que su análisis se realiza exclusivamente con los resultados del año 1999.

Normalmente, la pregunta sobre suficiencia educativa se completa con otras dos, también subjetivas: suficiencia de formación (conocimientos y habilidades ganados en el puesto laboral) y adecuación de los conocimientos y las habilidades al puesto de trabajo (si lo que se sabe es útil para el puesto de trabajo o no). Estas dos preguntas se repiten en ambos cuestionarios, a diferencia de la primera, que, como se dijo, no está en el cuestionario del 2003. Nuestro interés recae ahora exclusivamente en la primera de las tres preguntas: la que inquiere por la suficiencia educativa.

La mitad de los entrevistados declaró, en 1999, que sus estudios eran más que suficientes para realizar su trabajo. En otras palabras: la mitad de la población ocupada se consideraba sobreeducada. Solamente una minoría (3,8 por ciento) contestó que sus estudios eran insuficientes. Apenas hay diferencias entre hombres y mujeres: dos puntos porcentuales a favor de éstas. Y lo que es más curioso, tampoco hay diferencias entre los tres grupos de edad. La sobreeducación subjetiva es muy parecida entre los jóvenes (53,9 por ciento), los adultos $(50)$ y los mayores $(48,3)$.

Otro dato de interés es que tampoco hay diferencias de ingresos entre las tres categorías de suficiencia educativa. Tienen parecida media de ingresos tanto los que estiman que sus estudios son insuficientes, como los suficientes o los más que suficientes: ganan algo menos los sobreeducados (los que tienen estudios más que suficientes); ganan algo más los ajustados (los que tienen estudios suficientes). Las diferencias, no obstante, no son grandes $(F=5.021$; sign. $=0,000)$.

Tampoco parece haber relación entre la sobreeducación subjetiva y el criterio objetivo utilizado anteriormente elaborado a partir de los cinco estratos ocupacionales: el coeficiente de contingencia es muy bajo (véase la tabla 9).

Esta falta de explicación objetiva a la respuesta en cuestión hace dudar del interés de la misma. Da la sensación de que el entrevistado tiende a responder de forma estereotipada a una pregunta cuya respuesta hay que dar por supues-

Tabla 9. Ajuste educativo según criterio subjetivo y según estratos. Población ocupada española en 1999.

\begin{tabular}{lcccc}
\hline $\begin{array}{l}\text { Ajuste educativo } \\
\text { subjetivo }\end{array}$ & \multicolumn{2}{c}{$\begin{array}{l}\text { Ajuste educativo según estratos } \\
\text { Infraeducados }\end{array}$} & $\begin{array}{c}\text { Ajustados } \\
\text { Sobreeducados }\end{array}$ & Total \\
\hline Insuficiente & 0,9 & 2,5 & 0,4 & 3,7 \\
Suficiente & 9,1 & 29,6 & 6,8 & 45,4 \\
Más que suficiente & 8,5 & 32,7 & 9,6 & 50,8 \\
Total & 18,5 & 64,7 & 16,8 & 100 \\
\hline
\end{tabular}

Coeficiente de contingencia $=0,073$ (sig. $=0,000$ ). $\mathrm{V}$ de Cramer $=0,052 . N=5.631$.

Fuente: Encuesta de calidad de vida en el trabajo, 1999. Elaboración propia. 
ta. Forma parte de la opinión pública la idea de que existe sobreeducación y se responde siguiendo esa idea. O sea, la sobreeducación subjetiva no sirve como indicador de sobreeducación. Seguramente, la constatación de esta falta de interés es lo que ha llevado a los autores del cuestionario a prescindir de la misma en las ediciones posteriores.

\section{Conclusiones}

Pese a la larga tradición de estudio de la sobreeducación, la operativización del concepto sigue planteando problemas. Para facilitar nuestro trabajo, hemos elaborado una tipología simple de sobreeducación. Primero se ha distinguido entre la generalizada y la relativa. La generalizada es propia del proceso de expansión educativa que acompaña a la modernización y al desarrollo económico. Alcanza a toda o a casi toda la población. Constituye el marco teórico más general en el que debe entenderse la sobreeducación. Es el resultado de la expansión educativa y de la progresiva autonomía de la educación respecto a las demás variables de la estructura social (en particular, de la ocupación y de los ingresos). Desde este punto de vista, la sobreeducación no lo es tanto, ya que termina por diluirse y compensarse en el resto del paisaje social. Ahí radica la dificultad de la operativización del concepto. En la medida en que se asume por el sistema, no tiene por qué ser conflictiva.

La sobreeducación relativa puede llegar a ser conflictiva. Se entiende por la relativa aquél exceso de educación que posee una minoría en cada categoría ocupacional en comparación con el resto de población de esa categoría. En la medida en que se produce el desacoplamiento general entre educación y ocupación, siempre habrá sobreeducación relativa. Ahora bien, nos hemos visto obligados a distinguir entre la sobreeducación relativa discutible y la evidente. La aparente (el 16,4 por ciento de la población ocupada española en el 2003, según nuestros cálculos) se obtiene de acuerdo con la fórmula habitual de definir la sobreeducación (población con años de escolaridad por encima de una desviación estándar sobre la media en cada categoría ocupacional). Ahora bien, esta definición es discutible, porque la población correspondiente tiene ingresos más elevados que el resto de la población ocupada en la misma categoría ocupacional. No puede haber sobreeducación si el exceso educativo implica mayores ingresos. En este caso, la sobreeducación es rentable y, por lo tanto, no es tal.

La sobreeducación evidente intenta evitar ese inconveniente. Para ello, nos ponemos en el peor de los casos: población con el nivel educativo universitario y ocupada en los dos estratos ocupacionales inferiores (el 2,1 por ciento de la población ocupada española del 2003, según nuestros cálculos). En el año 2003, los universitarios de los estratos inferiores ganaban lo mismo, incluso menos, que el resto de ocupados en esos estratos. Por consiguiente, es un buen indicador de sobreeducación para ese año. Ahora bien, en la encuesta del 1999 no ocurría lo mismo: los universitarios de esos estratos ganaban más que el resto. 
Esto induce a pensar que la operativización del concepto es doblemente problemática: primero, porque se trabaja con realidades que a veces evolucionan muy deprisa, como es el caso de la expansión educativa a partir de la Ley Villar Palasí de 1970 o el incremento de la renta per capita a partir de la entrada en la Unión Europea (momento de ascenso de la difusión en forma de « $S$ »), $\mathrm{y}$, segundo, porque tratamos con elementos que actúan de forma sistémica (se compensan los cambios de un elemento con cambios en otro).

Estas pueden ser las razones que expliquen los dos resultados más importantes que hemos barajado. Primero, las formulas tradicionales de contabilizar la sobreeducación son discutibles, no sirven. En estos casos, sigue siendo rentable tener un nivel educativo superior a la media en cada categoría ocupacional. Segundo, la tasa de sobreeducación evidente en el 2003 es muy baja: el 2,1 por ciento de la población ocupada (3,2 por ciento entre los jóvenes, 2 por ciento entre los adultos y 1 por ciento en la población mayor). En 1999 era aún inferior: 1,5 .

La sobreeducación subjetiva tampoco da mucho de sí. La mitad de la población encuestada afirmaba tener estudios más que suficientes para realizar su trabajo. Se trata de una respuesta estereotipada, forma parte de la opinión pública más extendida. Se llega a esa conclusión, la de sospechar que es una respuesta estereotipada, ante la falta de relación de esa respuesta con otras variables, tales como edad y sexo.

\section{Bibliografía}

Alba-Ramírez, A. (1993). «Mismatch in the Spanish labor Market: Overeducation?». Journal of Human Resources, 27(2), p. 259-278.

Alba-RamíreZ, A.; BlázQueZ, M. (2003). «Types of Job Match, Overeducation and Labour Mobility in Spain». En: Büchel, F., De Grip, A.; Mertens, A. Overeducation in Europe. Cheltenham (UK): Edward Elgar, p. 65-92.

Battu, H.; Belfield, C. R.; Sloane, P. J. (2000). «Over-education: How sensitive are the measures?». National Institute Economic Review, 171, p. 82-93.

BÉDUWÉ, C.; Planas, J. (2002). Expansión educativa y mercado de trabajo. Estudio comparativo realizado en cinco paises europeos: Alemania, España, Francia, Italia, Reino Unido, con referencia a los Estados Unidos. Instituto Nacional de las Cualificaciones. Consultado en: <http://edex.univ-tlse1.fr/edex/>.

BerG, I. (1970). Education and Jobs: The Great Training Robbery. Nueva York: Praeger. Boudon, R. (1977). «Education and Social Mobility: A Structural Model». En: Karabel, J.; Halsey, H. (eds.). Power and Ideology in Education. Nueva York: Oxford University Press, p. 186-196.

Brunet ICART, I.; MORELl Blanch, A. (1998). «Mercado de trabajo y estrategias de valorización». Revista Española de Investigaciones Sociológicas, 82, p. 37-71.

BÜChel, F.; De GrIP, A.; MerTens, A. Overeducation in Europe. Cheltenham (UK): Edward Elgar.

CARABAÑA, J. (1999). Dos estudios sobre movilidad intergeneracional. Madrid: Fundación Argentaria y Visor.

CARABAÑA, J.; BuenO, C. (1996). Escalas de prestigio ocupacional. Madrid: CIS. 
CASTELlS, M. (2000). La era de la información: economía, sociedad y cultura. La sociedad red. Madrid: Alianza.

Collins, R. (1989). La sociedad credencialista. Madrid: Akal.

Durkheim, E. (1975). Educación y sociología. Barcelona: Península [1a ed. 1922].

EASTERLIN, R. A. (1981). "Why Isn't the Whole World Developed». Journal of Economics History, 41.

Fina Sanglas, L.; Toharia Cortés, L.; García Serrano, C.; Mañé Vernet, F. (2000). «Cambio ocupacional y necesidades educativas de la economía española». En: SÁEZ, F. (coord.). Formación y empleo. Madrid: Argentaria y Visor, p. 47-154.

Folger, J.; NAM, C. B. (1964). «Trends in education in relation to the occupational structure». Sociology of Education, 38, p. 19-33.

García Espejo, M. I. (1998). Recursos formativos e inserción laboral de los jóvenes. Madrid: CIS.

Garrido, L. (1991). Prospectiva de las ocupaciones y la formación en la España de los noventa. Madrid: Instituto de Estudios y Análisis Económicos.

Gobernado, R. (2003). «El valor de la educación en el empleo del sector privado». Papers, 69, p. 11-29.

Gobernado, R. (1996). «Los límites de la expansión educativa». Revista Española de Pedagogía, 205, p. 487-510.

Goody, J. (1989). The Logic of Writing and the Organization of Society. Cambridge: Cambridge University Press.

INE (1994). Panorámica social de España 1994. Madrid.

JovANOvic, B. (1979). «Job Matching and the Theory of Turnover». Journal of Political Economy, 87, 5, p. 973-989.

MÜLLER, D. K. (1992). «El proceso de sistematización: el caso de la educación secundaria en Alemania». En: MÜLleR, D. K.; RINGER, F.; SIMON, B. (comps.). El desarrollo del sistema educativo moderno. Madrid: Ministerio de Trabajo y Seguridad Social, p. 37-86.

NorTh, D. C. (1982). «The Theorical Tools of the Economic Historian». En: Kindelberger, C. P.; Di Tella, G. Economics in the Long Vew. Essays in Honour of W.W. Rostow. Nueva York: Nueva York University Press.

RAMíREZ, O.; BOLI, J. (1999). "La construcción política de la escolarización de masas: sus orígenes europeos e institucionalización mundial». En: M. F. ENGUITA. Sociología de la educación. Lecturas básicas y textos de apoyo. Barcelona: Ariel, p. 297-314.

REIS, J. (1993). «El analfabetismo en Portugal en el siglo XIX: una interpretación». En: NuÑEZ, C. E.; TORTELLA, G. La maldición divina. Ignorancia y atraso económico en perspectiva histórica. Madrid: Alianza, p. 237-270.

RINGER, F. (1992). «La segmentación en los modernos sistemas educativos europeos: el caso de la educación secundaria en Francia entre 1865 y 1920». En: MÜLlER, D. K; RINGER, F.; SiMON, B. (comps.). El desarrollo del sistema educativo moderno. Madrid: Ministerio de Trabajo y Seguridad Social, p. 87-130.

RuBB, S. (2003). «Overeducation in the labour market: A comment and re-analysis of a meta-analysis». Economics of Education Review, 22, p. 621-629.

Rubinson, R.; BROWNe, I. (1994). «Education and Economy». En: SMELSER, N. J.; Swedberg, R. (eds.). The Handbook of Economic Sociology. Princenton y Nueva York: Princenton University ress y Russell Sage Foundation, p. 581-599.

Ruesga Benito, A. M.; García De La Cruz, J. M.; Murayama Rendon, C. (2000). «Introducción: la relación entre educación y empleo. Una aproximación a su aná- 
lisis económico». En: SÁEZ, F. (coord.). Formación y empleo. Madrid: Argentaria y Visor, p. 19-46.

Rumberger, R. (1981). Overeducation in the U.S. Labor Market. Nueva York: Praeger. Rumberger, W. R. (1999). «El impacto potencial de la tecnología en la demanda de cualificación para los empleos futuros». En: ENGUITA, M. F. Sociología de la educación. Barcelona: Ariel, p. 105-130.

San Segundo Gómez; Petrongolo, B. (2000). «¿Estudias o trabajas? Los efectos del desempleo sobre la escolarización». En: SÁEZ, F. (coord.). Formación y empleo. Madrid: Argentaria y Visor, p. 421-450.

SANCHEZ CARRIÓN, J. J. (1996). Manual de análisis de datos. Madrid: Alianza.

Sicherman, N.; GALOR, O. (1990). «A Theory of Career Mobility». Journal of Political Economy, 98, 1, p. 160-192.

SloAne, P. J. (2003). «Much ado About Nothing? What does the Overeducation Literature Really Tell us?». En: BÜCHEL, F., DE GrIP, A.; MerTENS, A. Overeducation in Europe. Cheltenham (UK): Edward Elgar, p. 11-48.

SNYDERS, G. (1978). Escuela, clase y sociedad. Madrid: Alberto Corazón.

STEEDMAN, H. (1992). «Instituciones determinantes: las endowed grammar schools y la sistematización de la educación secundaria inglesa». En: MÜLLER, D. K.; RINGER, F.; Simon, B. (comps.). El desarrollo del sistema educativo moderno. Madrid: Ministerio de Trabajo y Seguridad Social, p. 161-194.

Thurow, L. C. (1977). Education and Economic Equality». En: KARABEL, J.; HalSEY, H. (eds.). Power and Ideology in Education. Nueva York: Oxford University Press, p. 325-334.

Veblen, T. (1974). Teoría de la clase ociosa. Méjico: FCE [1a ed. 1889]. 